\title{
Penoscrotal extramammary Paget's disease: surgical techniques and follow-up experiences with thirty patients
}

\author{
Qi Chen*, Yan-Bo Chen*, Zhong Wang, Zhi-Kang Cai, Yu-Bing Peng, Da-Chao Zheng, Li-Ming Ma, Hai-Jun Yao \\ and Juan Zhou
}

To report the surgical management, complications and prognosis of patients with penoscrotal extramammary Paget's disease (EMPD) at different clinical stages. Between 2003 and 2008, a total of 30 male patients with penoscrotal EMPD were enrolled and evaluated. All enrolled subjects received frozen biopsy-guided local wide resection and immediate reconstruction. Patients were followed every 3 months postoperatively. Among the 30 patients who accepted and underwent frozen biopsy-guided local wide resection treatment and reconstruction, two $(6.7 \%)$ cases exhibited positive margins, verified by pathological examination, and underwent re-excision after surgery. The technique of primary closure or an adjacent flap was used in $10(33.3 \%)$ cases, split-thickness skin grafts were used in 15 $(50 \%)$, and an anterolateral thigh perforator flap was used in five cases $(16.7 \%)$. The postoperative complications were acceptable. The mean follow-up time was $64.9 \pm 29.6$ months. Of all 30 cases, 22 patients $(73.3 \%)$ survived with no evidence of recurrence, four patients (13.3\%) exhibited local recurrence, two patients $(6.7 \%)$ exhibited both local recurrence and distant metastasis and the remaining two patients $(6.7 \%)$ exhibited distant metastasis. Five patients died from metastasis or cachexia. Current surgical techniques, including primary closure, adjacent flaps, split-thickness skin flaps and anterolateral thigh perforator flaps are able to reconstruct all types of defects with acceptable complications. Some patients with negative margins went on to exhibit local recurrence, potentially due to adnexal carcinoma or internal malignancy.

Asian Journal of Andrology (2013) 15, 508-512; doi:10.1038/aja.2013.27; published online 20 May 2013

Keywords: frozen biopsy; Paget's disease; penoscrotal reconstruction; surgical excision

\section{INTRODUCTION}

Extramammary Paget's disease (EMPD) is an uncommon tumour that typically occurs in the vulva and perianal regions. The involvement of the male genitalia in this disease is less frequently reported, with most cases occurring in Asia. ${ }^{1-3}$ The treatment of penoscrotal EMPD includes surgical excision, chemotherapy, radiotherapy, laser treatment and imiquimod cream. ${ }^{4-8}$ However, local wide excision and immediate reconstruction is the preferred treatment of Chinese urologists. ${ }^{9-11}$

Our experience with 130 patients evaluated at mid-term follow-up demonstrated that intraoperative frozen-section biopsy was a reliable means to detect invisible tumours and could significantly reduce recurrence. ${ }^{3}$ When we used this technique to completely resect the lesions and preserve normal tissues, we encountered the challenge of reconstructing the different sizes and shapes of postoperative skin defects (Figures $\mathbf{1 b}, \mathbf{2 b}, \mathbf{3 b}$ and $\mathbf{4 b}$ ). In this report, we introduce our current surgical techniques and the follow-up outcomes of our experience treating penoscrotal EMPD.

\section{PATIENTS AND METHODS}

\section{Clinical data}

This study was performed at the Urology Department of our hospital. The internal review board and ethical committee of the hospital approved the study protocol, and all patients provided written informed consent.

From 2003 to 2008, 30 male patients with lesions located in the penoscrotal area were diagnosed with EMPD by pathological biopsy and were treated in our urology department. Ten were diagnosed in our hospital, and the other 20 cases were transferred from other medical centres in China after the pathological diagnosis.

All patients were assessed by physical examination, ultrasonography of the internal organs, chest X-ray, pelvic computed tomography (CT) and serum tumour markers (prostate-specific antigen, $\alpha$-fetoprotein and carcinoembryonic antigen). Two patients exhibited concomitant visceral cancer (one with prostatic cancer and the other with gastric cancer).

The routine follow-up included physical examinations, biopsy of suspicious local recurrence (every 3 months), chest X-ray and pelvic CT (every 6 months).

\section{Staging system}

No formal staging system has been accepted for penoscrotal EMPD. In this study, we used the modified staging criteria proposed by Shutze et al. ${ }^{12}$ for perianal disease:

(I) disease in the epidermis without visceral or adnexal carcinoma;

(II) penoscrotal EMPD associated with adnexal carcinoma or a visceral carcinoma; 

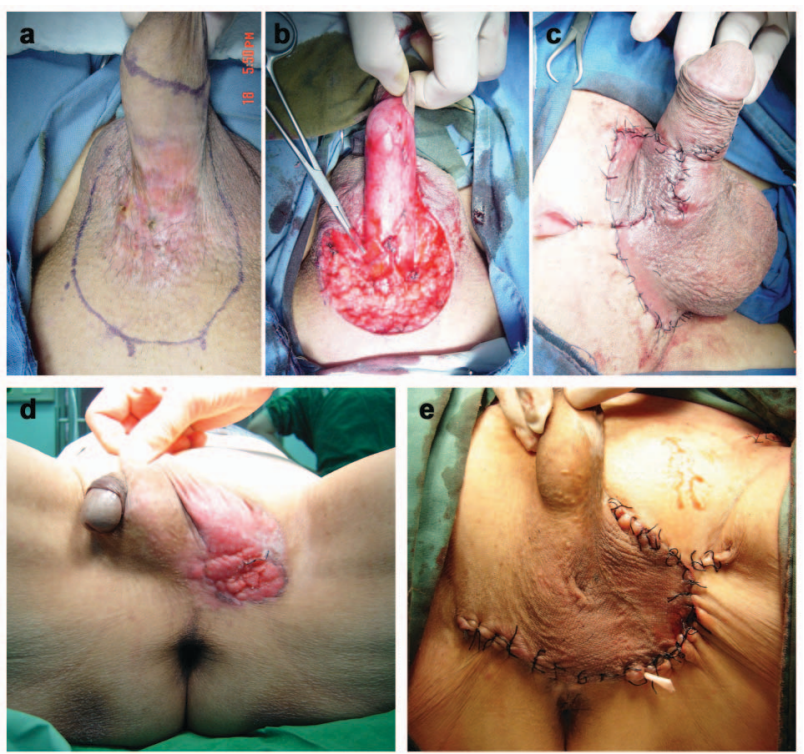

Figure 1 The frozen biopsy was prepared (a). Scrotal skin was used to cover the wound directly (d and $\mathbf{e}$ ), or scrotal skin was used as a random flap to cover the wound ( $\mathbf{b}$ and $\mathbf{c}$ ).

(III) penoscrotal EMPD with involved regional lymph nodes;

(IV) penoscrotal EMPD with distant metastasis.

\section{Frozen biopsy-guided local wide resection}

Since January 2003, we have used a frozen biopsy procedure that was designed for the treatment of penoscrotal EMPD at our centre. As shown in Figure 1a, a boundary designated as the 'clock face' is inscribed $2 \mathrm{~cm}$ from the lesion with a marking pen. On the edge of the clock face, six points are taken for frozen-section analysis. If one of the points is positive for malignant tissue, additional biopsies are taken on an extended boundary of the clock face $2 \mathrm{~cm}$ away from the positive
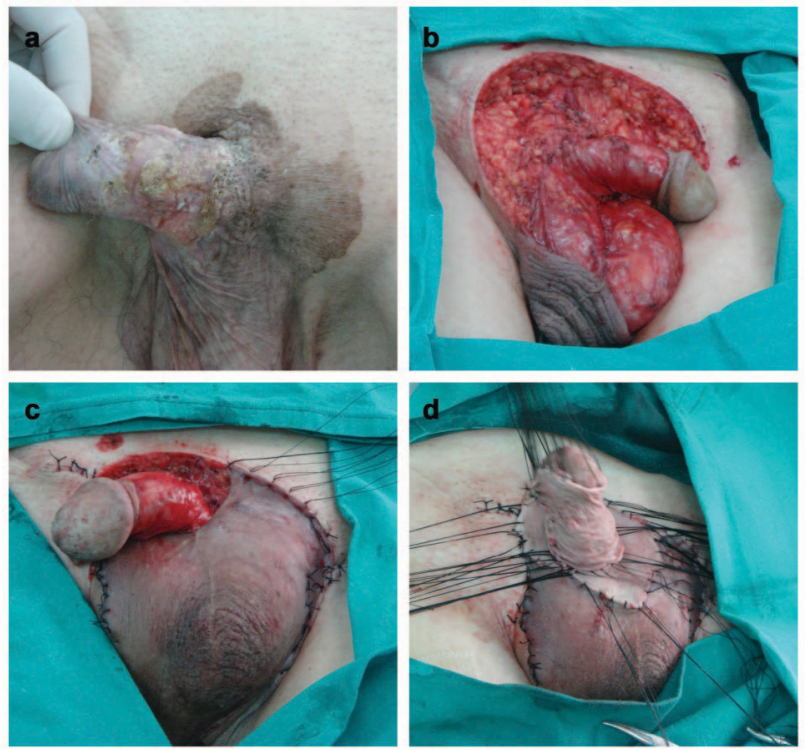

Figure 2 The diseased region was large (a), and the lesion size increased after resection (b). The wound was minimized by scrotal flaps (c) and covered with split-thickness skin grafts (d).
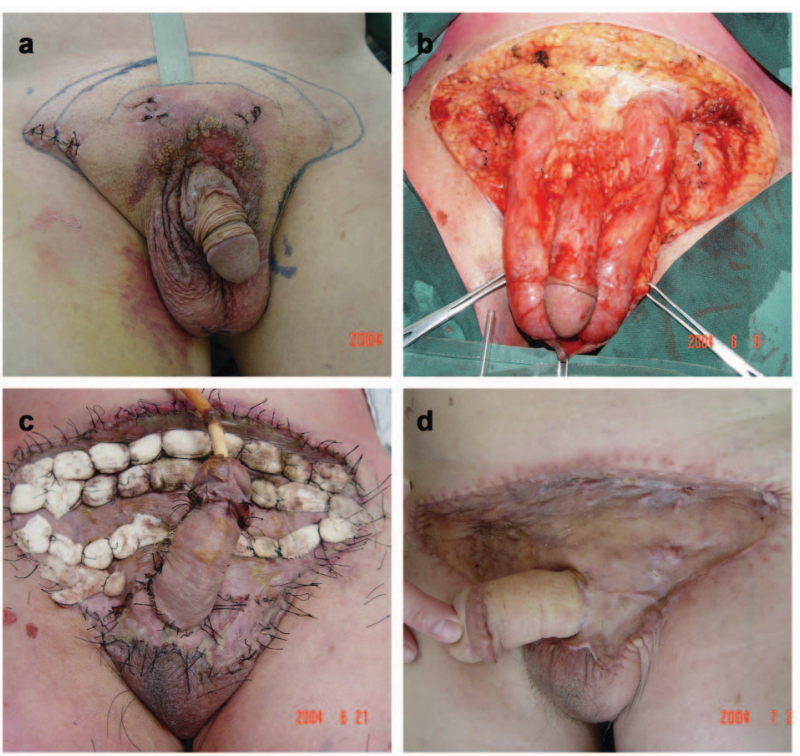

Figure 3 A frozen biopsy is shown that is positive for malignant tissue at the red points and negative at the green points (a). The lesion was covered by total splitthickness skin grafts ( $\mathbf{b}$ and $\mathbf{c}$ ). The result was satisfactory one month after the surgery (d).

point (Figure 3a). When all biopsy points are negative for malignant tissue, the final boundary is determined for resecting the lesion. For small lesions, we took six points for a frozen biopsy, while for large lesions, we took 12 or more.
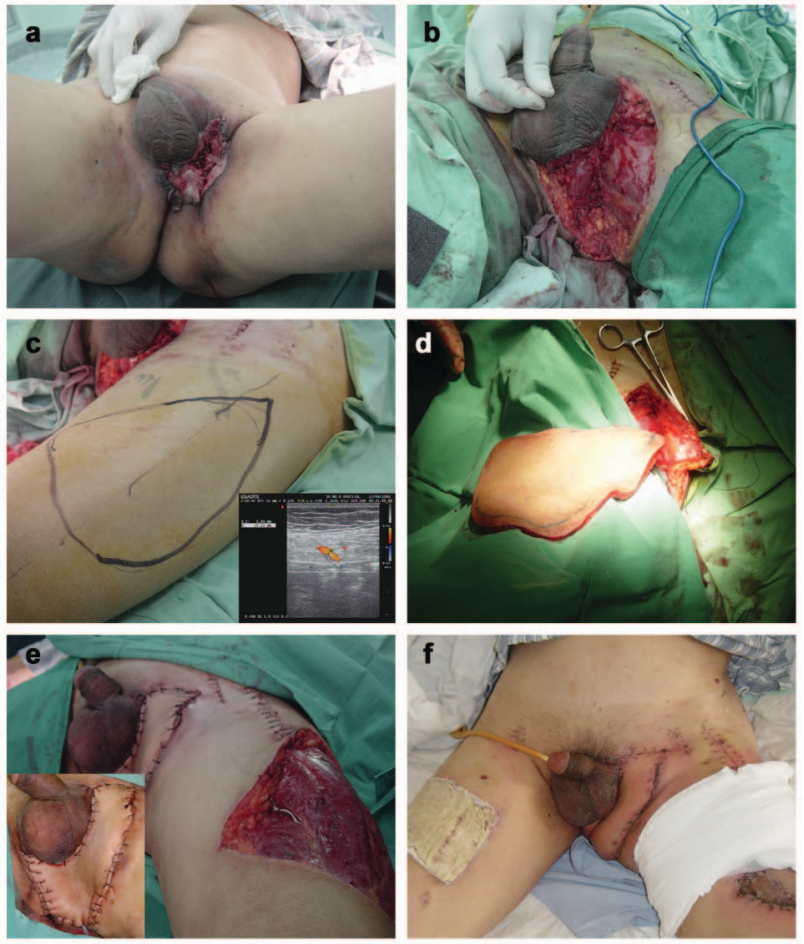

Figure 4 One case exhibited a huge wound and an ulcer in the perineum (a). The lesion was both large and deep after resection (b). The lateral femoral vessels were probed by Doppler ultrasound, and an anterolateral thigh perforator flap was designed (c and $\mathbf{d}$ ). The wound was well-covered by this flap (e) and healed well (f). 


\section{Enlarged inguinal lymph nodes}

If enlarged inguinal lymph nodes were palpable by physical examination or detected by ultrasonography, the sentinel lymph nodes were resected for histology to detect metastasis. If positive, lymph node dissection was performed (Figure 3a and $\mathbf{3 b}$ ). During lymph node dissection, the superficial and deep nodes were removed bilaterally.

\section{Surgical techniques for reconstruction}

Primary closure or adjacent flap. Adjacent flaps include the low abdominal rotary flaps and the scrotal flaps. Some cases included smaller lesions. After resection, by virtue of the flexibility of scrotal skin, the scrotal flap could be used effectively to suture the wound directly or as a random flap to cover the wound (Figure 1). Low abdominal rotary flaps were able to minimize defects or to cover wounds in the pubic area directly.

Adjacent flaps plus split-thickness skin grafts or total split-thickness skin grafts. Most patients exhibit a large wound after local wide resection. In some cases, we were able to make use of low abdominal rotary flaps and scrotal flaps to minimize the wound. Afterwards, the reduced wound was typically covered with split-thickness skin grafts (Figure 2). In other cases, the lesions were large, and rotational or adjacent flaps were difficult to obtain. In these cases, we used total split-thickness skin grafts to repair the skin defect (Figure 3). The detailed techniques of split-thickness skin grafts have been previously described. ${ }^{3}$

Anterolateral thigh perforator flap. In misdiagnosed patients or those who receive improper treatment for an extended period of time, the lesions can be large and deep (Figure 4a). After total resection of these lesions, the split-thickness skin graft barely covered the wide and rugged defect (Figure 4b). In these cases, we used the anterolateral thigh perforator flap technique to repair the defect.

First, a connection line was made from the anterior superior iliac spine to the outer edge of the patella. An area was established using the midpoint of the connection line was the centre of a circle $3 \mathrm{~cm}$ in radius. We used colour Doppler ultrasound to probe this area to identify the skin perforator pedicle of the lateral femoral vessels (Figure 4c). The flap was then mapped based on these vessels. Ideally, the designed flap dimension should be $10 \%$ larger than the defect. The anatomical details of the vasculature of this flap have been described by Sananpanich et al..$^{13}$

Second, the vasculature was anatomized to an appropriate length to ensure that the flap exhibited sufficient movement (Figure 4d). A tunnel was made between the donor area and the defect. The flap was squeezed through this tunnel to cover the wound (Figure 4e). The donor area was repaired by split-thickness skin grafts from the other thigh. The postoperative view of a patient is shown in Figure $4 \mathbf{f}$.

\section{RESULTS}

\section{Perioperative data}

All 30 patients with EMPD accepted and received local wide excision treatment, which included gastrectomy in one patient and prostatectomy in a second patient because their EMPD was accompanied by gastric cancer and prostatic cancer, respectively. The patients' characteristics and perioperative data are presented in Table 1. Most patients (20 cases, 67.7\%) received a pathological diagnosis and underwent an operation within 12 months. However, six patients had been misdiagnosed and had received improper treatment for more than 18 months before being appropriately treated.
Table 1 Perioperative data of clinical stage and surgery

\begin{tabular}{lc}
\hline Clinical data & \\
\hline Age, year, mean (range) & $67.6 \pm 10.4(52-90)$ \\
Mean interval of disease before surgery & \\
$\quad$ Month, mean (range) & $13.3 \pm 12.1(3-61)$ \\
Clinical stage, number (\%) & \\
I & $15(50 \%)$ \\
II & $9(30 \%)$ \\
III & $6(20 \%)$ \\
Surgical techniques, number (\%) & \\
Primary closure or adjacent flap & $10(33.3 \%)$ \\
Split-thickness skin grafts & $15(50 \%)$ \\
Anterolateral thigh perforator flap & $5(16.7 \%)$ \\
Resected skin size*, cm², mean (range) & $93.7 \pm 189.2(8-750)$ \\
Operative time, min, mean (range) & $145.4 \pm 87.2(38-395)$ \\
Hospital stay, day, mean (range) & $12.5 \pm 1.6(3-25)$ \\
\hline
\end{tabular}

*Resected skin size was calculated by Image-Pro Plus software (version 6.0; Media Cybernetics, Silver Spring, MD, USA).

According to our biopsy procedure, 13 of 30 (43.3\%) patients exhibited positive frozen biopsy results on the first lateral boundary, which was $2 \mathrm{~cm}$ from the lesion. Therefore, additional biopsies were needed to confirm the final margin during the operation. After surgery, however, two $(6.7 \%)$ cases exhibited positive lateral margins verified by pathological examination. These two patients underwent re-excision 2 weeks later.

We used three surgical techniques to repair and reconstruct tissue defects. The technique of primary closure or adjacent flap was used in $10(33.3 \%)$ cases, split-thickness skin grafts were used in 15 cases $(50 \%)$ and anterolateral thigh perforator flaps were used in five cases (16.7\%). There were no perioperative mortalities.

Enlarged inguinal lymph nodes were detected in 10 patients who then underwent lymph node resection. Six of these patients exhibited positive pathological results and underwent inguinal lymph node dissection. Patients with positive nodes received adjuvant chemotherapy.

\section{Complications}

Complications were classified as early or late (Table 2). Of 15 patients in the split-thickness skin grafts group, 4 patients exhibited various degrees of necrosis $(5 \%-15 \%$ of the size of the grafted area) of the grafted skin. Necrosis was attributed to wound infection, subcutaneous effusion or poor blood supply. The wounds were kept clean and monitored for granulation repair. One patient in the anterolateral thigh perforator flap group complained of paroxysmal neuralgia and skin numbness of the lower limb postoperatively. We attributed this to

Table 2 Early and late surgical complications

\begin{tabular}{lc}
\hline Complications & No./cases (\%) \\
\hline Early (<30 days after surgery) & \\
Wound infection & $5 / 30(16.7 \%)$ \\
Necrosis of grafted skin & $4 / 15(26.7 \%)$ \\
Necrosis of perforator flap & $0 / 5(0 \%)$ \\
Subcutaneous effusion or hematoma & $3 / 30(10 \%)$ \\
Injury of cutaneous nerve of thigh & $1 / 30(3.3 \%)$ \\
Deep venous thrombosis & $1 / 30(3.3 \%)$ \\
Lymphatic leakage & $1 / 6(16.7 \%)$ \\
Late (>30 days after surgery) & \\
Lymphedema & $1 / 6(16.7 \%)$ \\
Sexual dysfunction & $8 / 19(42.1 \%)$ \\
Scar contracture & $4 / 30(13.3 \%)$ \\
\hline
\end{tabular}


intraoperative injury of the cutaneous nerve of the thigh. After inguinal lymph node dissection, lymphatic leakage occurred in one patient in the early phase, and lymphedema of the lower limb occurred in another patient in late phase. Management of lymphatic leakage included prolonged drainage until the leakage resolved. Treatment of lymphedema included a lower limb compression bandage, diuretics and antibacterial prophylaxis.

Prior to the surgery, 19 patients reported being sexually active. However, eight patients experienced sexual dysfunction postoperatively. Of the eight cases, six were attributed to removing too much penile skin. Loss of skin sensation could be the predominant cause. We attempted to a use small dose of vardenafil to improve their symptoms. These patients remain in follow-up. The international index of erectile function (IIEF-5) was used to evaluate sexual function.

\section{Recurrent and survival data}

By June 2012, the patients had been followed for a mean of $64.9 \pm 29.6$ months (34-107 months). Of the 30 cases (two cases exhibited positive margins in the first surgery and underwent re-excision 2 weeks later) whose surgical margins were clear, 22 patients (73.3\%) survived with no evidence of recurrence, four patients (13.3\%) exhibited local recurrence within 2 years (two for stage I and two for stage II), two patients $(6.7 \%)$ exhibited both local recurrence and distant metastasis (bone for stage II and lung for stage III) within three years, and the remaining two patients $(6.7 \%)$ exhibited distant metastases (brain and bone, both in stage III) within 3 years. Five patients (two with stage II disease and three with stage III disease) died from metastasis or cachexia, and two patients died from cancer-unrelated causes (one from myocardial infarction and one from stroke). Thus far, the prognosis remains positive for stage I lesions and worse for stage II and stage III lesions.

\section{DISCUSSION}

EMPD is a rare neoplasm that usually occurs in elderly male patients. The primary treatment is surgical excision. ${ }^{7,9,11,14-17}$ Non-surgical methods are also used but only in a few cases. Hata reported that radiotherapy was a curative therapy in 22 patients with EMPD. ${ }^{18}$ Photodynamic therapy is believed by some authors to be effective. ${ }^{19-20}$ Imiquimod 5\% cream was successfully used in two series. ${ }^{8,21}$ The efficacy of carbon dioxide laser treatment remains open to question. $^{6,22}$ After extended research and exploration, our institution established a standard surgical procedure for penoscrotal EMPD, which includes a staging system, frozen biopsy protocol, current techniques of repair and reconstruction and standard follow-up methods.

\section{Diagnosis}

EMPD diagnosis is often delayed because this uncommon disease is initially considered by most doctors to be a benign condition. In this study, the mean interval of disease before surgery was more than 13 months. We concluded that the following reasons primarily explained the delay of correct treatment. First, the initial clinical signs of penoscrotal EMPD were nonspecific features, including erythema, eczema, erosion and pruritus. Therefore, penoscrotal EMPD can often be misdiagnosed as penoscrotal eczema or dermatitis. Second, even if doctors recognize the misdiagnosis, they are unsure whether to perform a biopsy. Moreover, some patients are subconsciously unwilling to undergo biopsy. Third, EMPD can be hard to distinguish from Bowen's disease and melanoma by pathological diagnosis. ${ }^{23}$ The pathological characteristics of Paget cells have been detailed previously. ${ }^{3}$

\section{Surgical treatments}

Inguinal lymph node enlargement might be due to inflammation or tumour metastasis. If metastasis is suspected, a lymph node biopsy should be performed. Based on the biopsy results, we decide whether lymph node dissection should be undertaken. In our experience, if infection of the wound is suspected, lymph node dissection should be delayed two to three months after treatment of the primary lesions. This can reduce the infection and the incidence of lymphatic leakage.

In recent years, the major methods for reconstructing penoscrotal defects after local wide excision include split-thickness skin graft, local scrotal flaps, rotary flaps and primary closure. ${ }^{9,11,14}$ In this report, we introduce the 'anterolateral thigh perforator flap' technique to reconstruct defects after the resection of penoscrotal EMPD lesions. The anterolateral thigh flap is an effective option for the reconstruction of soft-tissue defects such as those in the head, neck, extremities, perineal and scrotal areas. ${ }^{24,25}$ The anterolateral thigh flap exhibits many advantages, including a large cutaneous area, long vascular pedicle, adjustable sickness and acceptable donor-site morbidity. ${ }^{13}$ In this study, of the five patients treated with anterolateral thigh flaps, no severe complications occurred, and only one patient complained of paroxysmal neuralgia and skin numbness of the lower limb postoperatively.

\section{Margins and recurrence}

During surgery, the major techniques used to confirm the resection margin include Mohs micrographic surgery, fluorescent dyes and frozen biopsy. ${ }^{3,10,26,27}$ Some reports demonstrate that clear margins are highly relevant to whether local recurrence occurs. In the report presented by Zhang et al., ${ }^{28}$ all five cases of local recurrences involved positive margins and received a second surgery. In the report of $\mathrm{Xu}$ et al., ${ }^{29}$ nine cases exhibited recurrence (seven local and two distant), and five of these cases exhibited positive margins. Additionally, another report of five cases with negative margins exhibited no local recurrence with a mean 20 months of follow-up. ${ }^{11}$ However, data from other studies demonstrate different results. Although these methods could eventually ensure clear margins, local recurrence persisted. Lee et al. ${ }^{1}$ reported that the local recurrence rate was $10 \%$ for Mohs micrographic surgery versus 30\% for frozen section plus wide excision. Most of the recurrence cases exhibited negative margins. In the study reported by Yoon et al. ${ }^{27}$ using the fluorescent dye method, no correlation was observed between the status of lateral resection margins and local recurrence. Of the 28 patients, 23 with clear lateral resection margins exhibited an unexpectedly high local recurrence rate (five of 23, 21.7\%). A similar situation occurred in the study of Fishman et al..$^{30}$ In a report of vulva EMPD, the margin state was not predictive of local recurrence, as $33 \%$ of cases with negative margins and $40 \%$ of cases with positive margins exhibited disease recurrence. In our previous report, patients with positive margins exhibited a high rate of tumour recurrence $(56 \%) .^{3}$

In this study, two patients with positive margins underwent reexcision and exhibited no local recurrence. Nevertheless, local recurrence was observed in six patients $(20 \%)$ who exhibited clear margins: two in stage I, three in stage II and one in stage III. Most stage I cases $(86.7 \%)$ with clear margins did not recur. Thus, we conclude that positive margins are the primary factor causing local recurrence in stage I cases. Further, adnexal carcinoma and internal malignancy play an important role. Our assumption supports the two current models of EMPD pathogenesis. In the first theory, Paget's cells arise from the epidermis and extend into the contiguous epithelium of hair follicles and eccrine sweat ducts. ${ }^{31}$ The second theory indicates that disease results from an adnexal carcinoma or an internal malignancy that subsequently spreads into the contiguous epidermis of the skin. ${ }^{32,33}$ 
However, for the two stage I cases with clear margins that recurred, we propose that EMPD might arise from a multifocal origin or intraepithelial metastasis, which may explain why local recurrence can occur after the entire margin was confirmed to be negative and in the absence of internal malignancy. A larger prospective study with a longer follow-up may provide more evidence.

\section{CONCLUSION}

This series demonstrates that local wide excision and immediate reconstruction represents a preferred treatment for penoscrotal EMPD. Current surgical techniques are able to reconstruct many types of defects with acceptable complications. However, patients with negative margins can exhibit local recurrence, potentially due to adnexal carcinoma or internal malignancy.

\section{AUTHOR CONTRIBUTIONS}

QC, YBC, ZW and ZKC designed the study and performed the sugeries. YBP, DCZ and LMM performed the statistical analysis. QC and YBC drafted the manuscript. QC, HJY and JZ completed the patients' follow-up data.

\section{COMPETING FINANCIAL INTERESTS}

All authors declare that there are no competing financial interests.

\section{ACKNOWLEDGMENTS}

This study was supported by grants from the Natural Science Foundation of China (No. 81070544), the Shanghai Science and Technology Project (No. 08140901700) and the Science and Technology Fund of Shanghai JiaoTong University School of Medicine (No. 09XJ21002).

1 Lee KY, Roh MR, Chung WG, Chung KY. Comparison of Mohs micrographic surgery and wide excision for extramammary Paget's Disease: Korean experience. Dermatol Surg 2009; 35: 34-40.

2 Hatta N, Yamada M, Hirano T, Fujimoto A, Morita R. Extramammary Paget's disease: treatment, prognostic factors and outcome in 76 patients. Br J Dermatol 2008; 158: 313-8.

3 Wang Z, Lu M, Dong GQ, Jiang YQ, Lin MS et al. Penile and scrotal Paget's disease: 130 Chinese patients with long-term follow-up. BJU Int 2008; 102:485-8.

4 Mochitomi Y, Sakamoto R, Gushi A, Hashiguchi T, Mera K et al. Extramammary Paget's disease/carcinoma successfully treated with a combination chemotherapy: report of two cases. J Dermatol 2005; 32: 632-7.

5 Luk NM, Yu KH, Yeung WK, Choi CL, Teo ML. Extramammary Paget's disease: outcome of radiotherapy with curative intent. Clin Exp Dermatol 2003; 28: 360-3.

6 Becker-Wegerich PM, Fritsch C, Schulte KW, Megahed M, Neuse W et al. Carbon dioxide laser treatment of extramammary Paget's disease guided by photodynamic diagnosis. Br J Dermatol 1998; 138: 169-72.

7 Lai CS, Lin SD, Yang CC, Chou CK. Surgical treatment of the penoscrotal Paget's disease. Ann Plast Surg 1989; 23: 141-6.

8 Gil-Bistes D, Kluger N, Rigau V, Guillot B. Successful treatment of recurrent extramammary Paget's disease of the penis and scrotum with imiquimod $5 \%$ cream. Presse Med 2011; 40: 1205-6.
9 Lai YL, Yang WG, Tsay PK, Swei H, Chuang SS et al. Penoscrotal extramammary Paget's disease: a review of 33 cases in a 20-year experience. Plast Reconstr Surg 2003; 112: 1017-23.

10 Zhu Y, Ye DW, Chen ZW, Zhang SL, Qin XJ. Frozen section-guided wide local excision in the treatment of penoscrotal extramammary Paget's disease. BJU Int 2007; 100: 1282-7.

11 Yang Q, Yu L, Wang L, Gao L, Su M et al. Rotary mutiflaps for defect reconstruction in penoscrotal extramammary Paget's disease. Urol Oncol 2008; 26: 600-3.

12 Shutze WP, Gleysteen JJ. Perianal Paget's disease. Classification and review of management: report of two cases. Dis Colon Rectum1990; 33: 502-7.

13 Sananpanich K, Tu YK, Kraisarin J, Chalidapong P. Flow-through anterolateral thigh flap for simultaneous soft tissue and long vascular gap reconstruction in extremity injuries: anatomical study and case report. Injury 2008; 39: 47-54.

14 Park S, Grossfeld GD, McAninch JW, Santucci R. Extramammary Paget's disease of the penis and scrotum: excision, reconstruction and evaluation of occult malignancy. J Urol 2001; 166: 2112-6.

15 Por YC, Tan BK, Hong SW, Chia SJ, Cheng CW et al. Use of the scrotal remnant as a tissue-expanding musculocutaneous flap for scrotal reconstruction in Paget's disease. Ann Plast Surg 2003; 51: 155-60.

16 Yang WJ, Kim DS, Im YJ, Cho KS, Rha KH et al. Extramammary Paget's disease of penis and scrotum. Urology 2005; 65: 972-5.

17 Macedo A Jr, Fichtner J, Hohenfellner R. Extramammary Paget's disease of the penis. Eur Urol 1997; 31: 382-4.

18 Hata M, Omura M, Koike I, Wada H, Miyagi E et al. Role of radiotherapy as curative treatment of extramammary Paget's disease. Int J Radiat Oncol Biol Phys 2011; 80: $47-54$

19 Kitagawa KH, Bogner P, Zeitouni NC. Photodynamic therapy with methylaminolevulinate for the treatment of double extramammary Paget's disease. Dermatol Surg 2011; 37: 1043-6.

20 Raspagliesi F, Fontanelli R, Rossi G, Ditto A, Solima E et al. Photodynamic therapy using a methyl ester of 5 -aminolevulinic acid in recurrent Paget's disease of the vulva: a pilot study. Gynecol Oncol 2006; 103: 581-6.

21 Berman B, Spencer J, Villa A, Poochareon V, Elgart G. Successful treatment of extramammary Paget's disease of the scrotum with imiquimod 5\% cream. Clin Exp Dermatol 2003; 28: 36-8.

22 Puppala S. Failure of carbon dioxide laser treatment in three patients with penoscrotal extramammary Paget's disease. BJU Int 2001; 88: 986-7.

23 Reed W, Oppedal BR, Eeg Larsen T. Immunohistology is valuable in distinguishing between Paget's disease, Bowen's disease and superficial spreading malignant melanoma. Histopathology 1990; 16: 583-8.

24 Wei FC, Jain V, Celik N, Chen HC, Chuang DC et al. Have we found an ideal soft-tissue flap? An experience with 672 anterolateral thigh flaps. Plast Reconstr Surg 2002; 109: 2219-26.

25 Yu P, Sanger JR, Matloub HS, Gosain A, Larson D. Anterolateral thigh fasciocutaneous island flaps in perineoscrotal reconstruction. Plast Reconstr Surg 2002; 109: 610-6.

26 Wagner RF Jr, Cottel WI. Treatment of extensive extramammary Paget disease of male genitalia with Mohs micrographic surgery. Urology 1988; 31: 415-8.

27 Yoon SN, Park IJ, Kim HC, Yu CS, Lee MW et al. Extramammary Paget's disease in Korea: its association with gastrointestinal neoplasms. Int J Colorectal Dis 2008; 23: 1125-30.

28 Zhang N, Gong K, Zhang X, Yang Y, Na Y. Extramammary Paget's disease of scrotumreport of 25 cases and literature review. Urol Oncol 2010; 28: 28-33.

29 Xu K, Fang Z, Zheng J, Lu Y, Li B et al. Intraoperative frozen biopsy in wide surgical excision of Paget's disease of the scrotum. Urol Oncol 2009; 27: 483-5.

30 Fishman DA, Chambers SK, Schwartz PE, Kohorn EI, Chambers JT. Extramammary Paget's disease of the vulva. Gynecol Oncol 1995; 56: 266-70.

31 Lloyd J, Flanagan AM. Mammary and extramammary Paget's disease. J Clin Pathol 2000; 53: 742-9.

32 Marchesa P, Fazio VW, Oliart S, Goldblum JR, Lavery IC et al. Long-term outcome of patients with perianal Paget's disease. Ann Surg Oncol 1997; 4: 475-80.

33 Sarmiento JM, Wolff BG, Burgart LJ, Frizelle FA, Ilstrup DM. Paget's disease of the perianal region—an aggressive disease? Dis Colon Rectum 997; 40: 1187-94. 